\title{
An Improved Reflection Colorimeter Integrated with a Coaxial Optical-fiber Cable for Highly Sensitive Solid-phase Colorimetry Using a Membrane Filter
}

\author{
Hitoshi Mizuguchi, ${ }^{* 1 \dagger}$ Yusuke TaniguchI, ${ }^{* 1}$ Suherman Suherman, ${ }^{* 2}$ Kinichi Morita, ${ }^{* 3}$ \\ Toshio TAKAYANAGI, ${ }^{* 1}$ and Yasutada SUZUKI ${ }^{* 4}$ \\ *1 Department of Applied Chemistry, Graduate School of Science and Technology, Tokushima University, \\ Tokushima 770-8506, Japan \\ *2 Department of Chemistry, Faculty of Mathematics and Natural Sciences, Universitas Gadjah Mada, \\ Yogyakarta 55281, Indonesia \\ *3 Ushio Inc., 1-6-5 Marunouchi, Chiyoda, Tokyo 100-8150, Japan \\ *4 College of Bioscience and Chemistry, Kanazawa Institute of Technology, 3-1 Yatsukaho, Hakusan, \\ Ishikawa 924-0838, Japan
}

\begin{abstract}
Highly sensitive solid-phase colorimetry for nickel ion was demonstrated using an improved reflection colorimeter equipped with a coaxial optical-fiber cable. The nickel complex with $\alpha$-furil dioxime was collected on a small-size membrane filter embedded in a disposable syringe filter unit. The leading edge of the optical-fiber cable was connected to the syringe filter unit via a Luer-lock fitting, and the color intensity of the sample on the filter was evaluated accurately. The detection limit was $0.8 \mathrm{ng}$ in $2.5 \mathrm{~mL}$ of the complex solution $\left(0.3 \mu \mathrm{g} \mathrm{L} \mathrm{L}^{-1}\right)$. This improved configuration is applicable to highly sensitive on-site analysis without expensive instruments nor high laboratory skills.
\end{abstract}

Keywords Reflection colorimeter, optical fiber, nickel, $\alpha$-furil dioxime, simple analytical method

(Received October 22, 2020; Accepted December 4, 2020; Advance Publication Released Online by J-STAGE December 11, 2020)

\section{Introduction}

The development of simple, rapid, and inexpensive test methods for the determination of harmful elements is essential to control water quality for environmental protection and a safe drinkingwater supply. Spectrophotometry is the most frequently used for screening purposes, however, these methods generally lack sensitivity to detect those elements around the WHO's guideline levels. Numerous techniques, such as solvent extraction ${ }^{1}$ and solid-phase extraction, ${ }^{2}$ to enrich target analytes and clean up the sample were investigated for a long time to increase the sensitivity and selectivity. Further developments are continued to fulfill various analytical needs. ${ }^{3,4}$

The membrane filter is one of the most promising media for enriching and separating trace substances from complex matrices. ${ }^{5,6}$ The target analytes are concentrated on a membrane filter by simple filtration after appropriate derivatization using chromogenic reagents. ${ }^{7-10}$ This technique, which does not use harmful organic solvents, is suitable for on-site work. A direct comparison of the membrane filter colorations provided by the enrichment onto the filter surface enables a highly sensitive determination of the target analyte, ${ }^{11-25}$ and some methods are commercially available. However, most of these strategies are conducted by visual comparisons as simple analytical methods. Despite the excellent analytical performances, accurate evaluations of the color intensity are still dependent on relatively

$\dagger$ To whom correspondence should be addressed.

E-mail: mizu@ tokushima-u.ac.jp expensive instruments, such as a densitometer or a spectrophotometer, usually used for color measurements of solid samples.

The previous report showed a portable reflection-colorimeter consisted of a light-emitting diode (LED) as a light source, a photodiode as a light detector, and a coaxial optical-fiber cable. ${ }^{24}$ The LED's light illuminated on the membrane filter surface through the emitter fiber positioned at the center of the coaxial optical-fiber cable. The reflected light was then collected by the receiver fibers at the emitter fiber's circumference and detected by a photodiode. This configuration provides the ability to evaluate the color intensity of membrane filters and enables onsite work using a lightweight and compact body.

To improve the sensitivity with a smaller sample solution, it is more advantageous to concentrate the target analyte in a narrow area on a membrane filter. In this case, the incident light should be focused on the colored area to increase the signal of the reflection absorption and reduce the interference by light scattering on the surface out of the colored area. One of the reasonable solutions to this problem is fixing the fiber end-face close to the membrane filter surface. In the current study, a portable reflection-colorimeter was improved by integrating the leading edge of the coaxial optical-fiber cable and a Luer-lock fitting. A disposable syringe filter unit with a female Luer-lock fitting is adopted to enrich the target analyte in a small area $\left(0.06 \mathrm{~cm}^{2}\right)$ and attached to the reflection colorimeter to evaluate the color intensity accurately. In the discussion below, a highly sensitive determination of nickel is demonstrated using the currently proposed configuration. 

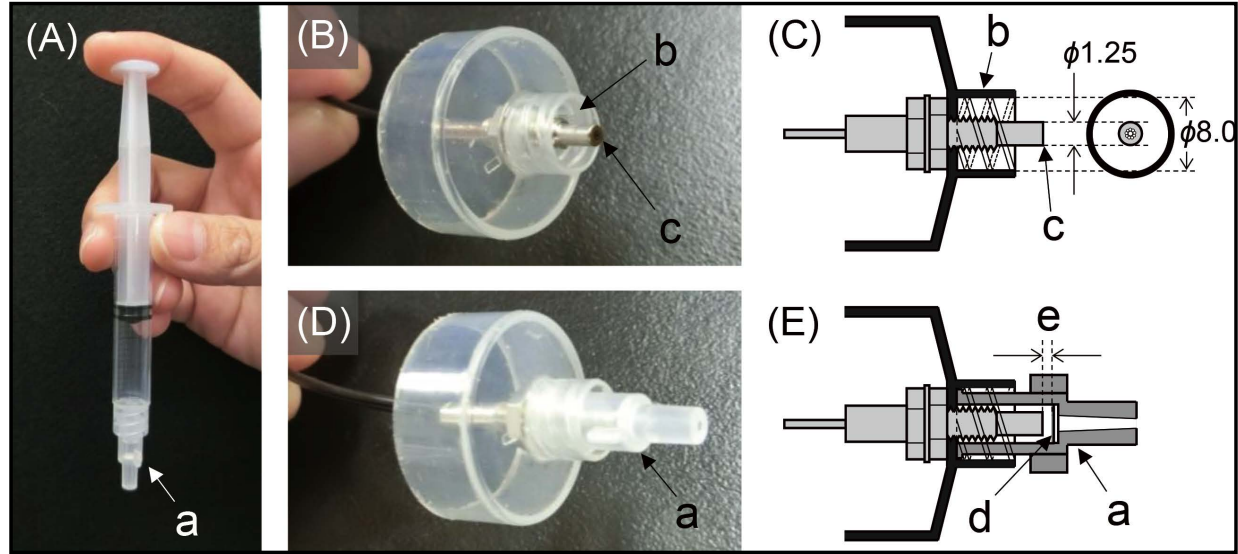

Fig. 1 Outline of the currently proposed configuration. (A) During filtration, (B, C) a leading head of the optical-fiber cable integrated with Luer-lock fitting, (D, E) after attaching a syringe filter unit for the color intensity measurement. Syringe filter unit (a), Luer-lock fitting (b), end face of the coaxial optical-fiber cable (c), embedded membrane filter (d), the working distance (e).

\section{Experimental}

\section{Reagents and materials}

A nickel standard solution $(1000 \mathrm{mg} / \mathrm{L})$ was purchased from Kanto Chemical Co. (Tokyo, Japan). The reagent, $\alpha$-furil dioxime $(\alpha$-FD), was obtained from Tokyo Chemical Industry Co., Ltd. (Tokyo, Japan) and dissolved in ethanol to make a $0.1 \mathrm{wt} \%$ solution. The $\mathrm{pH}$ buffer agent, $\mathrm{N}$-tris(hydroxymethyl)methyl-3-aminopropanesulfonic acid (TAPS), was obtained from Dojindo Laboratories (Kumamoto, Japan) and dissolved it in a $\mathrm{NaOH}$ aqueous solution to make a $0.1 \mathrm{M}$ buffer solution $(\mathrm{pH}$ 8.2). All other reagents used were of guaranteed reagent grade. All solutions were prepared using $18 \mathrm{M} \Omega \mathrm{cm}$ deionized water, which was obtained with a water-purification system (Milli-Q ${ }^{\circledR}$ Gradient A10, Millipore). A disposable syringe filter unit integrated with a membrane filter made of cellulose acetate (03CP045AN, an effective filtration area: $0.06 \mathrm{~cm}^{2}$, pore size: $0.45 \mu \mathrm{m}$ ) was purchased from ADVANTEC (Tokyo, Japan).

\section{Apparatus}

A portable reflection colorimeter integrated with a coaxial cable with optical fibers was constructed based on a previous report. $^{24}$ In this study, we used a white LED (NSPW500GS-K1, Nichia Co., Japan) and a Si photodiode (S2386-18K, Hamamatsu Photonics K. K., Japan) as a light source and a light detector, respectively. The coaxial cable (FD-G500, Panasonic Co., Japan) included receiver fibers at the circumference of a centerpositioned emitter fiber. The ends of the cable's bifurcated side were connected to the portable reflection colorimeter toward the LED and the photodiode, respectively. The leading edge of the other side of the coaxial cable was integrated with a Luer-lock fitting, which was obtained by cutting a plastic syringe and removing the tube tip beforehand (Fig. 1). The working distance, defined as the distance between the end-face of the optical fiber cable and the membrane filter surface, was adjusted with a nut tightened behind the Luer-lock fitting. The reflection absorbance $(A)$ was defined by the following equation,

$$
A=-\log \left(X / X_{0}\right)
$$

where $X$ and $X_{0}$ are the intensities of reflection light from the colored membrane filter and the reagent blank, respectively.
The measurements were conducted after optimizing the LED luminescence intensity to avoid photodiode output saturation.

\section{Typical procedure}

The preparation of complex solutions was the same as described in a previous report. ${ }^{17,25}$ To a $10-\mathrm{mL}$ aliquot of a sample solution containing nickel(II) ion, $1.0 \mathrm{~mL}$ of a $\alpha$-FD solution, $1.5 \mathrm{~mL}$ of a $\mathrm{pH}$ buffer solution, and $0.5 \mathrm{~mL}$ of a $5 \mathrm{M}$ sodium nitrate solution were added and filled up to $25 \mathrm{~mL}$ with water. In analyses for the river-water samples, $1.0 \mathrm{~mL}$ of a $1.0 \mathrm{M}$ sodium thiosulfate solution and $1.5 \mathrm{~mL}$ of a $0.6 \mathrm{M}$ sodium fluoride solution as masking agents were added before adding the $\alpha$-FD solution. After standing for $10 \mathrm{~min}$ at room temperature, a $2.5-\mathrm{mL}$ aliquot of the mixture was aspirated into a TERUMO syringe. Then, a syringe filter unit was installed to the syringe tip via a Luer-lock fitting, and filtration was performed manually. After drying the membrane filter, the filter unit was attached to a coaxial optical-fiber cable via a Luer-lock connection, and the reflection absorbance was measured to evaluate the color intensity of the membrane filter.

\section{Results and Discussion}

\section{Benefits of the proposed method}

The proposed method features the combination of the enrichment on a small-size membrane filter and an accurate evaluation of the color intensity using an improved handheld colorimeter. The enrichment of the target analyte to a smaller region of the membrane filter brings about a superior sensitivity with a smaller volume of the sample solution. Considering the on-site work, filtration using a plastic syringe is more suitable than another way, such as motorized aspiration and centrifuging, because of its simplicity and cost-effectiveness. On the other hand, integrating the leading edge of the coaxial optical-fiber cable to a Luer-lock fitting provided a fixed working distance between the membrane filter surface and the optical fiber endface. The incident light was easily focused on the coloration area only by attaching the filter unit. This configuration brought about superior sensitivity and precision in measuring the reflection absorbance of the colored membrane filters. 


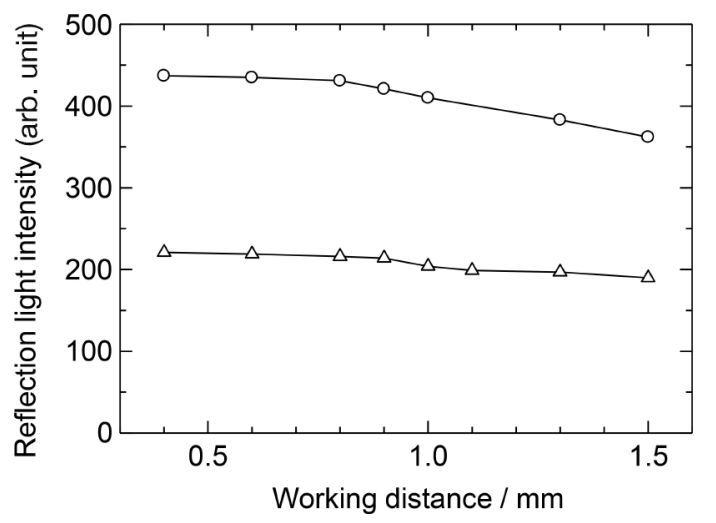

Fig. 2 Relationship between the working distance and the reflection light intensity. $\bigcirc$, Reagent blank; $\triangle$, containing $0.0625 \mu \mathrm{g}\left(25 \mu \mathrm{g} \mathrm{L}^{-1}\right)$ of nickel ion. The filtration volume was $2.5 \mathrm{~mL}$.

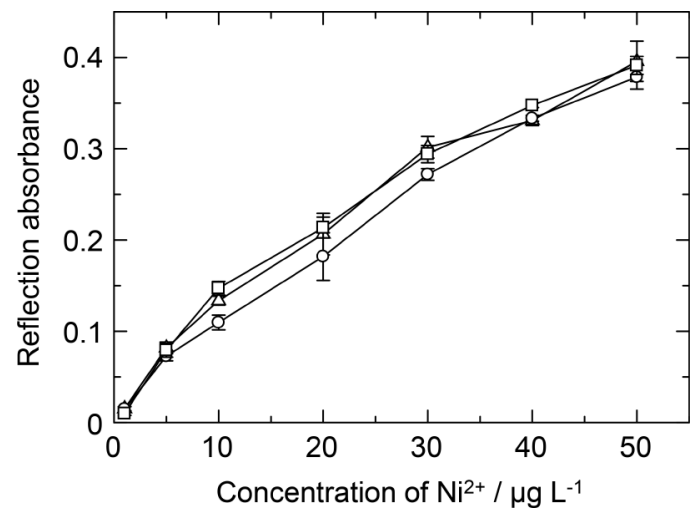

Fig. 3 Relationship between the concentration of nickel and the reflection absorbance. The working distances were $0.5(\triangle), 1.0(\bigcirc)$, and $1.5 \mathrm{~mm}(\square)$. The filtration volume was $2.5 \mathrm{~mL}$. The reflection absorption spectrum of the nickel complex collected on the membrane filter can be found in the supporting information.

\section{Effect of the working distance}

Figure 2 shows the effect of the working distance on the reflection light intensity. In this study, the working distance was measured by placing a caliper on the syringe filter unit's side, which was attached to the leading head of the optical-fiber cable via Luer-lock fitting. The intensity was gradually decreased with an increase in the working distance. In contrast, the reflection absorbance transition was almost the same among the different working distances when the reagent blank was used as a reference in each measurement (Fig. 3). This result shows that the sensitivity does not change in the working distance region of $0.5-1.5 \mathrm{~mm}$. However, the descent of the reflection light intensity derived from the longer working-distance tends to increase the minimum step of change in the reflection absorbance and raise the detection limit. Although this issue is dependent on the computing power of the embedded microcomputer, sufficient accuracy is obtained by working distance of $1.0 \mathrm{~mm}$ in the current specifications, as noted below.

\section{Effect of flow rate}

Figure 4 shows the effect of a flow rate against the reflection absorbance. The flow rate in the region from 0.5 to $1.5 \mathrm{~mL}$ $\mathrm{min}^{-1}$ was regulated using a syringe pump. The filtrations with

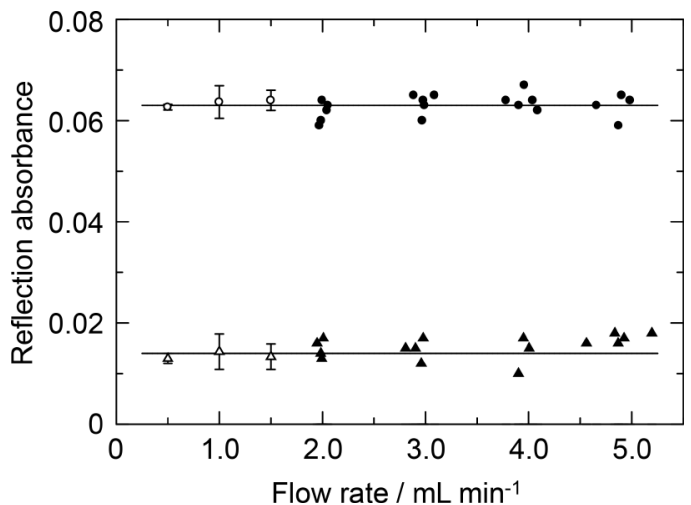

Fig. 4 Relationship between the reflection absorbance and the flow rate during filtration. The nickel concentrations were $1.0(\triangle, \mathbf{\Delta})$ and $5.0 \mu \mathrm{g} \mathrm{L}^{-1}(\mathrm{O}, \bullet)$, respectively. The filtration volume was $2.5 \mathrm{~mL}$. The flow rate in the region from 0.5 to $1.5 \mathrm{~mL} \mathrm{~min}^{-1}$ was controlled by a syringe pump $(\triangle, \bigcirc)$, whereas the filtrations at higher flow rates were conducted manually

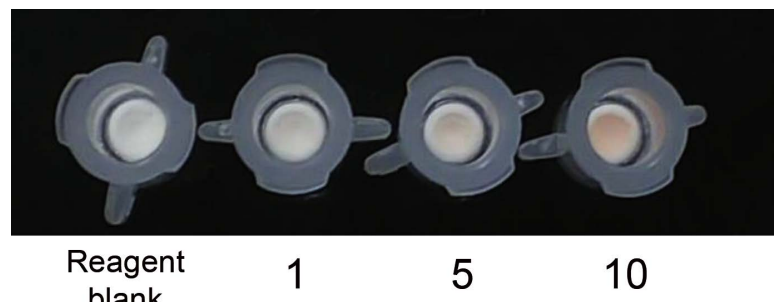

Concentration of $\mathrm{Ni}^{2+} / \mu \mathrm{L} \mathrm{L}^{-1}$

Fig. 5 Coloration of membrane filters in the syringe filter unit with relation to the nickel concentration. The filtration volume was $2.5 \mathrm{~mL}$.

higher flow rates were conducted manually, and the apparent flow rate was calculated from each filtration time measured using a stopwatch. The reflection absorbance was almost constant at flow rates from 0.5 to $5 \mathrm{~mL} \mathrm{~min}^{-1}$. This result shows that the reflection absorbance is not affected by the fluctuation of the flow rate, and this proposed method is suitable for manual operations in fieldwork.

\section{Calibration curve and detection limit}

The coloration of the membrane filter unit is shown in Fig. 5. The diameter of the colored area was $2.3 \mathrm{~mm}$. Although the colorations of the membrane filter less than $5.0 \mu \mathrm{g} \mathrm{L}^{-1}$ of nickel ion were hardly distinguished visually from the reagent blank, the color intensity was evaluated accurately by the proposed reflection colorimeter. The calibration curve assessed with the reflection absorbance was linear through the origin over a range of up to $25 \mu \mathrm{g} \mathrm{L}^{-1}$. The detection limit, defined as three-times the standard deviation, was $0.3 \mu \mathrm{g} \mathrm{L}^{-1}(0.8 \mathrm{ng})$, and the RSD value at 1.0 and $5.0 \mu \mathrm{g} \mathrm{L}^{-1}$ were 10.4 and $1.8 \% \quad(n=5)$, respectively. This analytical performance was comparable to the previously reported method using expensive instruments, such as a portable spectrophotometer ${ }^{17}$ or a densitometer. ${ }^{25}$

\section{Recovery test}

The analytical results, including recovery tests using river water, are summarized in Table 1. The certified sample used was NMIJ CRM 7202-c, provided by National Institute of 
Table 1 Analytical results of the river-water sample

\begin{tabular}{cccc}
\hline Sample & $\begin{array}{c}\text { Added amount/ } \\
\mu \mathrm{g} \mathrm{L}^{-1}\end{array}$ & $\begin{array}{c}\text { Found } \\
\mu \mathrm{g} \mathrm{L}^{-1}\end{array}$ & $\begin{array}{c}\text { Recovery, } \\
\%\end{array}$ \\
\hline River-water $^{\mathrm{a}}$ & 0 & $0.9 \pm 0.2$ & - \\
& 2.0 & $3.2 \pm 0.1$ & 113 \\
& 4.0 & $5.2 \pm 0.1$ & 106 \\
& 8.0 & $9.5 \pm 0.2$ & 107 \\
\hline
\end{tabular}

a. Certified reference material (NMIJ CRM 7202-c). The certified value of nickel content was $1.06 \pm 0.02 \mu \mathrm{g} \mathrm{kg}^{-1}$.

b. Three replicate analyses.

Advanced Industrial Science and Technology (AIST). There is a good agreement between the results obtained by the currently proposed method and the certified value. The recovery values were also acceptable for on-site analysis.

\section{Conclusions}

In this study, a reflection colorimeter equipped with a coaxial optical-fiber cable was improved by integrating with a Luerlock fitting to accurately evaluate the color intensity of a smallsize membrane filter embedded in a syringe filter unit. This combination enabled us to easily reproduce the constant working distance between the optical-fiber cable's end-face and the filter surface among measurements, and provided accurate analytical data without high laboratory skills. This configuration can apply to the previously developed colorimetric assays using membrane filters, and will provide useful protocols optimized for rapid, sensitive, and accurate analyses in field work.

\section{Supporting Information}

The reflection absorption spectrum of the nickel complex collected on the membrane filter is provided in supporting information. This material is available free of charge on the Web at http://www.jsac.or.jp/analsci/.

\section{References}

1. F. Pena-Pereira, I. Lavilla, and C. Bendicho, Spectrochim. Acta, Part B, 2009, 64, 1.
2. V. Camel, Spectrochim. Acta, Part B, 2003, 58, 1177.

3. K. Shigeta, Anal. Sci., 2019, 35, 1171.

4. C. Yeerum, P. I. N. Ayutthaya, K. Kesonkan, A. Chaiyakhan, and M. Vongboot, Anal. Sci., 2020, 36, 1137.

5. K. Goto and S. Taguchi, Anal. Sci., 1993, 9, 1.

6. S. Taguchi and K. Murai, Bunseki, 2008, 67.

7. K. Hiiro, T. Tanaka, A. Kawahara, M. Adachi, and K. Fukushi, Bunseki Kagaku, 1982, 31, E401.

8. K. Ohzeki, Y. Sato, Y. Kawamura, and T. Kambara, Bull. Chem. Soc. Jpn., 1983, 56, 2618.

9. S. Taguchi, E. Ito-oka, K. Masuyama, I. Kasahara, and K. Goto, Talanta, 1985, 32, 391.

10. J. Shida, H. Satake, N. Ono, and T. Fujikura, Talanta, 1990, 37,633 .

11. J. Shida and H. Takahashi, Anal. Sci., 1991, 7(Supplement), 675 .

12. J. Shida, H. Takahashi, and Y. Sakamoto, Talanta, 1994, 41, 143.

13. K. Yoshimoto, E. Kaneko, and T. Yotsuyanagi, Chem. Lett., 2000, 29, 6.

14. M. Hashimoto, N. Teshima, T. Sakai, and S. Kato, Bunseki Kagaku, 2005, 54, 783.

15. S. Taguchi, E. Seki, K. Murai, N. Hata, and H. Kuramitz, Bunseki Kagaku, 2006, 55, 525.

16. H. Mizuguchi, Y. Matsuda, T. Mori, A. Uehara, Y. Ishikawa, M. Endo, and J. Shida, Anal. Sci., 2008, 24, 219.

17. H. Mizuguchi, Y.-F. Zhang, H. Onodera, S. Nishizawa, and J. Shida, Chem. Lett., 2008, 37, 792.

18. T. Watanabe, H. Mizuguchi, and J. Shida, Bunseki Kagaku, 2011, 60, 339.

19. K. Murai, H. Honda, H. Okumura, and K. Okauchi, Bunseki Kagaku, 2011, 60, 507.

20. T. Funayama, H. Mizuguchi, and J. Shida, Bunseki Kagaku, 2013, 62, 685

21. H. Mizuguchi, Y. Shinoda, T. Wagatsuma, M. Takada, T. Kamijo, and J. Shida, Bunseki Kagaku, 2014, 63, 515.

22. T. Okazaki, H. Kuramitz, N. Hata, S. Taguchi, K. Murai, and K. Okauchi, Anal. Methods, 2015, 7, 2794.

23. H. Mizuguchi, R. Ishida, Y. Kouno, T. Tachibana, T. Honda, T. Kijima, and T. Takayanagi, Bunseki Kagaku, 2017, 66, 363.

24. Y. Hasegawa, Y. Suzuki, and S. Kawakubo, Anal. Sci., 2017, 33, 859.

25. H. Mizuguchi, R. Ishida, Y. Kouno, T. Tachibana, T. Honda, T. Kijima, Y. Yamamoto, and T. Takayanagi, Anal. Sci., 2018, 34, 907. 